\title{
Hidrogeomorfometria da microbacia do rio Cutia, Amazônia Sul-Ocidental, Brasil
}

\author{
Hydrogeomorphometry of the Cutia river microbasin, South-Western Amazon, Brazil \\ Hidrogeomorfometría de la microcuenca del río Cutia, Sur de la Amazonia Occidental, Brasil
}

Maria Larissa Hermido da Silva ORCID: https://orcid.org/0000-0002-5430-4312

Universidade Federal do Amazonas, Brasil

E-mail: mlarissahermido@gmail.com

Esther Cavalcante da Silva

ORCID: https://orcid.org/0000-0002-9994-5914

Universidade Federal do Amazonas, Brasil

E-mail: esther.scavalcante@gmail.com

Eduardo Ossamu Nagao

ORCID: https://orcid.org/0000-0002-0601-6906

Universidade Federal do Amazonas, Brasil

E-mail: eonagao@gmail.com

Wanderson Cleiton Schmidt Cavalheiro

ORCID: https://orcid.org/0000-0003-1356-8511

Cavalheiro Engenharia Rural e Empresarial Ltda, Brasil

E-mail: engflo.ro@gmail.com

Emanuel Maia

ORCID: https://orcid.org/0000-0002-5493-2183

Universidade Federal de Rondônia, Brasil

E-mail: emanuel@unir.br

Silfran Rogério Marialva Alves

ORCID: https://orcid.org/0000-0001-5220-234X

Universidade Federal do Amazonas, Brasil

E-mail: silfranrogerio@ufam.edu.br

Marta Silvana Volpato Sccoti

ORCID: https://orcid.org/0000-0001-5979-3218 Universidade Federal de Rondônia, Brasil E-mail: martasccoti@unir.br

Francisco Adilson dos Santos Hara

ORCID: https://orcid.org/0000-0002-3215-953X

Universidade Federal do Amazonas, Brasil

E-mail: fhara@ufam.edu.br

Jhony Vendruscolo

ORCID: https://orcid.org/0000-0003-3043-0581

Universidade Federal do Amazonas, Brasil

E-mail: jhonyvendruscolo@gmail.com

\begin{abstract}
Resumo
O planejamento da gestão dos recursos naturais é essencial para o desenvolvimento sustentável na região amazônica e tem como base as informações associadas às características da paisagem. Em face ao exposto, o presente trabalho tem como objetivo disponibilizar informações geométricas, topográficas e hidrográficas da microbacia do rio Cutia, a qual pertence a bacia do rio Guaporé, importante bacia do estado de Rondônia. Estas informações foram obtidas por meio de equações disponibilizadas na literatura e sensoriamento remoto, utilizando os softwares QGIS 2.10.1, TrackMaker e Google Earth, e imagens altimétricas do satélite ALOS (Sensor Palsar). A microbacia tem área de 19,20 km² de área, perímetro de $24,79 \mathrm{~km}$, forma alongada, baixa suscetibilidade a enchentes, altitudes de 261 a $346 \mathrm{~m}$, predominância de relevo suave ondulado $(47,45 \%), 90,36 \%$ da área apta a extremamente apta a mecanização agrícola e com baixa influência na propagação de incêndios, rede de drenagem com padrão dendrítico, $5^{a}$ ordem de drenagem, alta densidade de nascentes, alta densidade de drenagem, coeficiente de manutenção de $342,7 \mathrm{~m}^{2} \mathrm{~m}^{-1}$, canal principal divagante e baixo tempo de concentração. A microbacia do rio Cutia tem potencial para implantação de sistemas agropecuários, no entanto, são recomendadas práticas de manejo conservacionistas do solo e da água, para mitigar o impacto destes sistemas nos recursos naturais. Também são recomendados estudos sobre a distribuição espacial da vegetação nativa em áreas protegidas por lei na microbacia (reservas legais e áreas de preservação permanente), para auxiliar na delimitação de áreas prioritárias para a conservação dos recursos hídricos.
\end{abstract}

Palavras-chave: Geoprocessamento; Características fisiográficas; Recursos naturais; Planejamento e gestão ambiental. 


\begin{abstract}
Planning for the management of natural resources is essential for sustainable development in the Amazon region and is based on information associated with landscape characteristics. Given the above, the present study aims to provide geometric, topographic, and hydrographic information on the Cutia river microbasin, which belongs to the Guaporé river basin, an important basin in the state of Rondônia. This information was obtained through equations available in the literature and remote sensing, using the softwares: QGIS 2.10.1, TrackMaker and Google Earth, and altimetric images from the ALOS satellite (Sensor Palsar). The microbasin has an area of $19.20 \mathrm{~km}^{2}$, a perimeter of $24.79 \mathrm{~km}$, an elongated shape, low susceptibility to flooding, altitudes from 261 to $346 \mathrm{~m}$, predominance of smooth wavy relief (47.45\%), 90.36\% from the area apt to extremely apt to agricultural mechanization and with low influence on the spread of fires, drainage network with a dendritic pattern, 5th drainage order, high density of springs, high drainage density, maintenance coefficient of $342.7 \mathrm{~m}^{2} \mathrm{~m}^{-1}$, wandering main channel and low concentration time. The Cutia river microbasin has the potential to implement agricultural systems, however, soil and water conservation management practices are recommended to mitigate the impact of these systems on natural resources. Studies on the spatial distribution of native vegetation in areas protected by law in the microbasin (legal reserves and permanent preservation areas) are also recommended, to assist in the delimitation of priority areas for the conservation of water resources.
\end{abstract}

Keywords: Geoprocessing; Physiographic characteristics; Natural resources; Environmental planning and management.

\title{
Resumen
}

La planificación del manejo de los recursos naturales es fundamental para el desarrollo sostenible en la región amazónica y se basa en información asociada a las características del paisaje. Por lo anterior, el presente trabajo tiene como objetivo proporcionar información geométrica, topográfica e hidrográfica de la microcuenca del río Cutia. Esta información se obtuvo mediante ecuaciones disponibles en la literatura y teledetección, utilizando el software QGIS 2.10.1, TrackMaker y Google Earth, e imágenes altimétricas del satélite ALOS (Sensor Palsar). La microcuenca tiene un área de 19,20 $\mathrm{km}^{2}$, un perímetro de $24,79 \mathrm{~km}$, forma alargada, baja susceptibilidad a inundaciones, altitudes de 261 a $346 \mathrm{~m}$, predominio del relieve suave ondulado (47,45\%), 90,36\% del área apta para la mecanización agrícola y con poca influencia en la propagación de incendios, red de drenaje con patrón dendrítico, $5^{\circ}$ orden de drenaje, alta densidad de manantiales, alta densidad de drenaje, coeficiente de mantenimiento de $342,7 \mathrm{~m}^{2} \mathrm{~m}^{-1}$, canal principal errante y bajo tiempo de concentración. La microcuenca del río Cutia tiene el potencial de implementar sistemas agrícolas, sin embargo, se recomiendan prácticas de manejo de conservación del suelo y el agua para mitigar el impacto de estos sistemas en los recursos naturales. También se recomiendan estudios sobre la distribución espacial de la vegetación nativa en áreas protegidas por ley en la microcuenca (reservas legales y áreas de preservación permanente), para ayudar en la delimitación de áreas prioritarias para la conservación de los recursos hídricos.

Palabras clave: Geoprocesamiento; Características fisiográficas; Recursos naturales; Planificación y gestión ambiental.

\section{Introdução}

A microbacia é a menor parte da bacia hidrográfica (Cavalheiro \& Vendruscolo, 2019) e o seu tamanho permite obter informações detalhadas da paisagem com baixo custo financeiro e em tempo hábil, quando se utilizam geotecnologias (Soares, et al., 2019). As informações detalhadas da paisagem são desejadas para elaborar o planejamento ambiental e gerir adequadamente os recursos naturais, explicando, porque as microbacias são recomendadas (Vendruscolo et al., 2021a) e frequentemente utilizadas como unidades de gestão, conforme observado em trabalhos realizados no estado de Rondônia por Vendruscolo et al. (2021b), Souza et al. (2021), Silva et al. (2021), Donegá et al. (2021) e Santos et al. (2021).

O sensoriamento remoto e o Sistema de Informação Geográfica (SIG) são exemplos de geotecnologias (Florenzano, Lima \& Moraes, 2011) utilizadas para se obter informações detalhadas da paisagem. O sensoriamento remoto possibilita a aquisição de informações na superfície terrestre sem haver o contato direto com os objetos (Moreira, 2001). O SIG permite inserir e integrar em uma única base de dados, informações espaciais provenientes de dados cartográficos, dados censitários e cadastro urbano e rural, imagens de satélite, redes e modelos numéricos de terreno, e também oferecem ferramentas para combinar as informações, através de algoritmos de manipulação e análise, bem como para consultar, recuperar e visualizar o conteúdo da base de dados georreferenciados (Jucá, Carvalho \& Aguiar Júnior, 2006). Em função destas características o sensoriamento remoto e o SIG têm elevado potencial para aquisição de informações da paisagem na região amazônica.

A microbacia do rio Cutia está inserida na bacia do rio Guaporé, sub-bacia do rio Vermelho, e abrange aproximadamente 
37 estabelecimentos agropecuários privados (INCRA, 2018). Apesar da grande importância socioeconômica e ambiental, constata-se escassez de informações a respeito da paisagem desta microbacia na literatura. Portanto, o presente trabalho tem como objetivo utilizar geotecnologias para fornecer informações geométricas, topográficas e hidrográficas da microbacia do rio Cutia, com o intuito de auxiliar o planejamento e a gestão dos recursos naturais da região.

\section{Metodologia}

\subsection{Localização e características gerais da área de estudo}

A microbacia do rio Cutia está inserida na sub-bacia do rio Vermelho, localizada no município de Vilhena (Figura 1). Esta região tem clima Tropical do tipo Monção, temperaturas médias entre 24 e $26^{\circ} \mathrm{C}$ (Alvares et al., 2013), precipitação anual de 1.728,9 a 1.843,7 mm, concentrada nos meses de novembro a março (Franca, 2015), e solos classificados como Neossolos Quartzarênicos (SEDAM, 2002).

Figura 1. Localização da microbacia do rio Cutia, Amazônia Sul-Ocidental, Brasil.

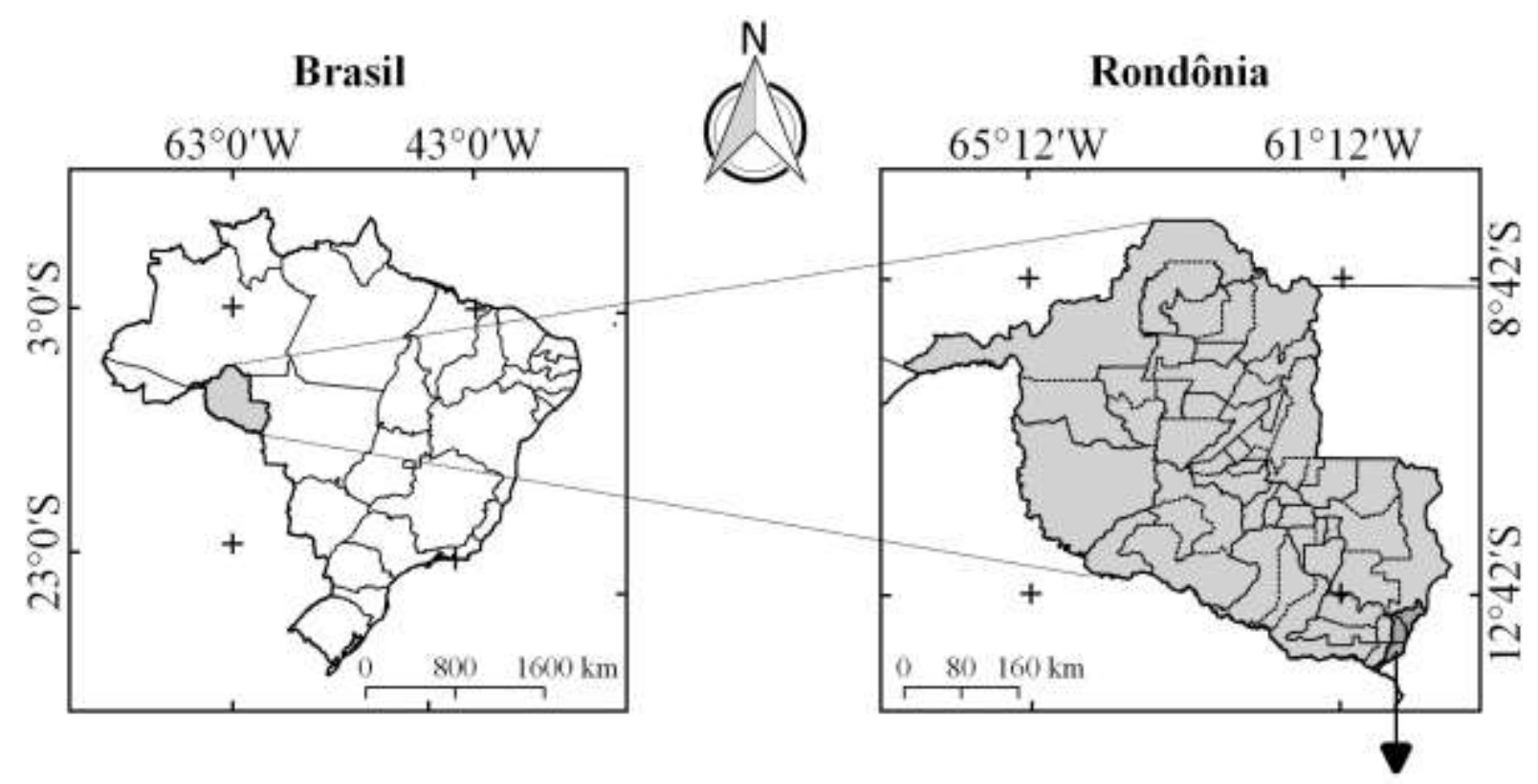

Microbacia do rio Cutia
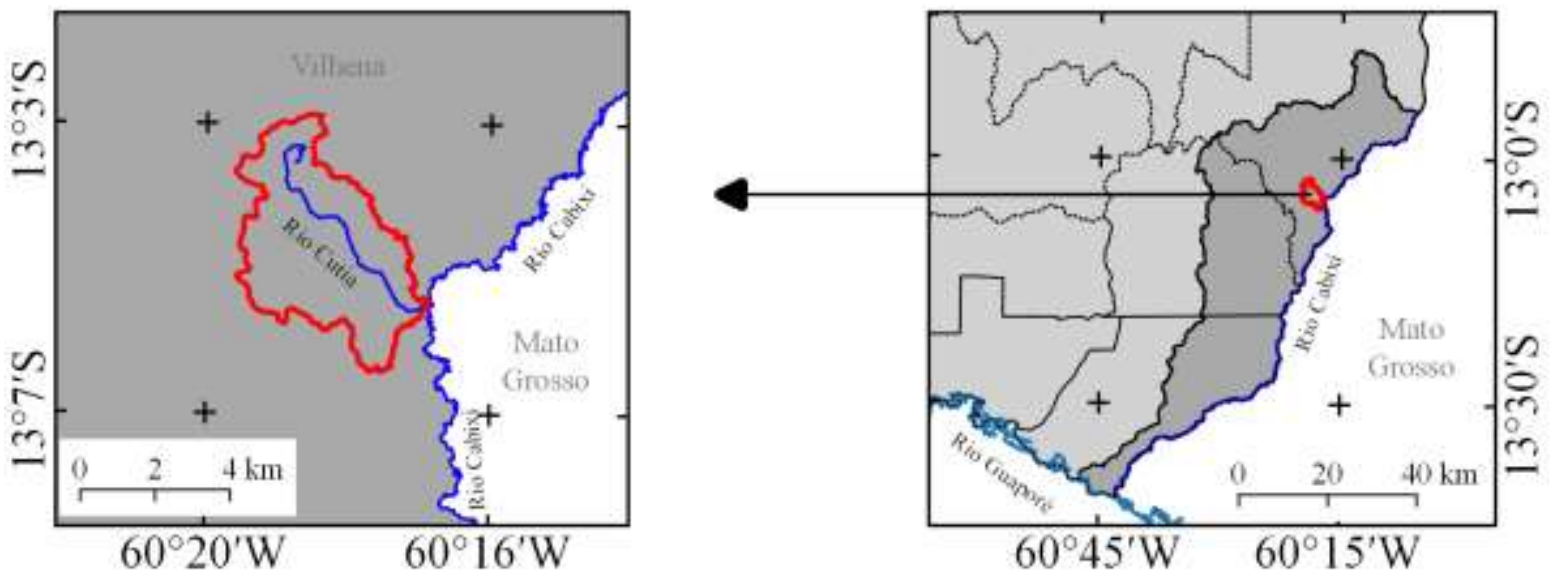

Sistema de Coordenadas Geográficas - Datum WGS 84

Fonte: Autores. 


\subsection{Características da paisagem}

Para a aquisição das características da paisagem e elaboração dos mapas, foram utilizados os softwares QGIS 2.10.1 (versão Pisa), Google Earth e TrackMaker Free (Versão 13.9.596), e imagens altimétricas do satélite ALOS (Sensor Palsar), com resolução espacial de 12,5 m (ASF, 2017). As metodologias são quantitativas e qualitativas, ao permitirem a identificação e análise das características da paisagem por meio da mensuração e interpretação dos valores (Pereira et al., 2018), e foi executada em quatro etapas, descritas detalhadamente a seguir:

\section{1 a Etapa - Características geométricas}

Para a delimitação do perímetro da microbacia foi usada a ferramenta TauDEM (passos: Pit Remove < D8 Flow Directions < D8 Contributing Area - $1^{\text {a }}$ versão $<$ Stream Definition By Threshold < Edição do ponto de exutório < D8 Contributing Area - $2^{\mathrm{a}}$ versão) e imagens altimétricas do satélite Alos (Sensor Palsar) (ASF, 2017), com resolução espacial de 12,5 m. O arquivo matricial, gerado no TauDEM, foi transformado para o formato vetorial, em seguida, dissolvido, suavizado e ajustado no software Google Earth, considerando as características da rede de drenagem e relevo. Após isso, foram calculados a área e o perímetro com a ferramenta "calculadora de campo".

Os parâmetros fator de forma, índice de circularidade e coeficiente de compacidade, foram calculados com as equações 1 (Villela \& Mattos, 1975), 2 (Christofoletti, 1980) e 3 (Villela \& Mattos, 1975), e comparados com dados da literatura (Tabela $1)$.

$$
F=\frac{A}{L^{2}}
$$

(Equação 1)

Onde: $\mathrm{F}$ = fator de forma; $\mathrm{A}=$ área da microbacia $\left(\mathrm{km}^{2}\right) ; \mathrm{L}=$ comprimento do eixo da microbacia $(\mathrm{km})$.

$$
\text { IC }=\frac{12,57 x A}{P^{2}}
$$

Onde: $\mathrm{Ic}=$ índice de circularidade; $\mathrm{A}=$ área da microbacia $\left(\mathrm{km}^{2}\right) ; \mathrm{P}=$ perímetro da microbacia $(\mathrm{km})$.

$$
K c=0,28 x \frac{P}{\sqrt{ } A}
$$

Onde: $\mathrm{Kc}=$ coeficiente de compacidade; $\mathrm{A}=$ área da microbacia $\left(\mathrm{km}^{2}\right) ; \mathrm{P}=$ perímetro da microbacia $(\mathrm{km})$.

Tabela 1. Classificação dos parâmetros geométricos: fator de forma, índice de sinuosidade e coeficiente de compacidade.

\begin{tabular}{ccl}
\hline Parâmetro & Limite & \multicolumn{1}{c}{ Classe } \\
\hline \multirow{2}{*}{ Fator de forma $^{1}$} & $<0,50$ & Não sujeito a enchentes \\
& $0,50-0,75$ & Tendência média a enchentes \\
& $0,76-1,00$ & Sujeito a enchentes \\
\hline Índice de circularidade $^{2}$ & $<0,51$ & Forma alongada \\
& $0,51-0,75$ & Forma intermediária \\
& $0,76-1,00$ & Forma circular \\
\hline \multirow{2}{*}{ Coeficiente de compacidade $^{1}$} & $1,00-1,25$ & Alta propensão a enchentes \\
& $1,26-1,50$ & Tendência média a enchentes \\
\hline
\end{tabular}

Fonte: ${ }^{1}$ Lima Júnior et al. (2012); ${ }^{2}$ Silva (2012).

\section{$2^{\text {a }}$ Etapa - Características topográficas}

As altitudes mínima e máxima foram obtidas diretamente da imagem altimétrica, e a altitude média foi mensurada com a ferramenta "estatística por zona". O relevo foi obtido com base na declividade do terreno, em seguida foi classificado em plano 
(0-3\%), suave ondulado (3-8\%), ondulado (8-20\%), forte ondulado (20-45\%) e montanhoso (45-50\%) (Santos et al., 2013). Para auxiliar nas análises da paisagem o relevo também foi classificado quanto à influência na propagação de incêndios e aptidão à mecanização agrícola (Tabela 2).

Tabela 2. Classificação da influência a propagação de incêndios e aptidão à mecanização agrícola em função da declividade.

\begin{tabular}{|c|c|c|}
\hline Parâmetro & Declividade (\%) & Classe \\
\hline \multirow{5}{*}{ Influência na propagação de incêndios ${ }^{1}$} & $\leq 15$ & Baixa \\
\hline & $16-25$ & Moderada \\
\hline & $26-35$ & Alta \\
\hline & $36-45$ & Muito alta \\
\hline & $>45$ & Extremamente alta \\
\hline \multirow{5}{*}{ Aptidão à mecanização agrícola ${ }^{2}$} & $0,0-5,0$ & Extremamente apta \\
\hline & $5,1-10,0$ & Muito apta \\
\hline & $10,1-15,0$ & Apta \\
\hline & $15,1-20,0$ & Moderadamente apta \\
\hline & $>20,0$ & Não apta \\
\hline
\end{tabular}

Fonte: ${ }^{1}$ Ribeiro et al. (2008); ${ }^{2}$ Höfig \& Araujo-Junior (2015).

\section{$3^{\text {a }}$ Etapa - Características hidrográficas}

Os rios foram gerados no software Google Earth, salvos no formato KML, e unidos no software TrackMaker para formar a rede de drenagem. Em seguida, foi identificado o padrão de drenagem por meio de uma análise visual, comparando a distribuição espacial da rede de drenagem da microbacia em estudo com a distribuição espacial de redes de drenagem de referência, disponibilizadas por Parvis (1950), e classificado a ordem dos rios de acordo com Strahler (1954).

Os parâmetros densidade de nascentes, densidade de drenagem, coeficiente de manutenção, índice de sinuosidade e tempo de concentração, foram calculados com as equações 4 (Christofoletti, 1980), 5 (Horton, 1932), 6 (Santos et al., 2012), 7 (Villela \& Mattos, 1975) e 8 (Kirpich 1940, apud Targa et al., 2012).

$$
D n=\frac{N}{A}
$$

Onde: $\mathrm{Dn}=$ densidade de nascentes $\left(\right.$ nascentes $\left.\mathrm{km}^{-2}\right) ; \mathrm{N}=$ número de nascentes; $\mathrm{A}=$ área da microbacia $\left(\mathrm{km}^{2}\right)$.

$$
D d=\frac{L}{A}
$$

Onde: $\mathrm{Dd}=$ densidade de drenagem $\left(\mathrm{km} \mathrm{km}^{-2}\right) ; \mathrm{L}=$ comprimento da rede de drenagem $(\mathrm{km}) ; \mathrm{A}=$ área da microbacia $\left(\mathrm{km}^{2}\right)$.

$$
C m=\frac{1}{D d} x 1000
$$

Onde: $\mathrm{Cm}=$ coeficiente e manutenção $\left(\mathrm{m}^{2} \mathrm{~m}^{-1}\right) ; \mathrm{Dd}=$ densidade de drenagem $\left(\mathrm{km} \mathrm{km}^{-2}\right)$.

$$
I s=\frac{L-D v}{L} \times 100
$$

Onde: Is = índice de sinuosidade $(\%) ; \mathrm{L}=$ comprimento do canal principal $(\mathrm{km}) ; \mathrm{Dv}=$ distância vetorial do canal principal $(\mathrm{km})$.

$$
T C=57 x\left(\frac{L^{3}}{H}\right)^{0,385}
$$

Onde: $\mathrm{Tc}=$ tempo de concentração (minutos); $\mathrm{L}=$ comprimento do talvegue principal $(\mathrm{km})$; $\mathrm{H}=$ desnível entre a parte mais elevada e a seção de controle (m). 
Os valores dos parâmetros ordem dos rios, densidade de nascentes, densidade de drenagem e índice de sinuosidade foram classificados de acordo com a literatura (Tabela 3).

Tabela 3. Classificação das características hidrográficas.

\begin{tabular}{|c|c|c|c|}
\hline Parâmetro & Unidade & Limite & Classe \\
\hline Ordem dos rios ${ }^{1}$ & Unidades & $\begin{array}{r}1-3 \\
4-6 \\
>6\end{array}$ & $\begin{array}{l}\text { Riachos pequenos } \\
\text { Riachos médios } \\
\text { Rios grandes }\end{array}$ \\
\hline Ordem dos rios ${ }^{2}$ & Unidades & $\begin{array}{c}1 \\
2 \\
3 \\
\geq 4\end{array}$ & $\begin{array}{l}\text { Improvável habitat de peixes } \\
\text { Baixas condições para habitação } \\
\text { Moderadas condições para habitação } \\
\text { Elevadas condições para habitação }\end{array}$ \\
\hline Densidade de nascentes ${ }^{3}$ & Nascentes $\mathrm{km}^{-2}$ & $\begin{array}{c}<3 \\
3-7 \\
7-15 \\
>15\end{array}$ & $\begin{array}{l}\text { Baixa } \\
\text { Média } \\
\text { Alta } \\
\text { Muito alta }\end{array}$ \\
\hline Densidade de drenagem ${ }^{4}$ & $\mathrm{~km} \mathrm{~km}^{-2}$ & $\begin{array}{c}<0,50 \\
0,50-2,00 \\
2,00-3,50 \\
>3,50\end{array}$ & $\begin{array}{l}\text { Baixa } \\
\text { Média } \\
\text { Alta } \\
\text { Muito alta }\end{array}$ \\
\hline Índice de sinuosidade ${ }^{5}$ & $\%$ & $\begin{array}{c}<20 \\
20-29 \\
30-39 \\
40-50 \\
>50\end{array}$ & $\begin{array}{c}\text { Muito reto } \\
\text { Reto } \\
\text { Divagante } \\
\text { Sinuoso } \\
\text { Muito sinuoso }\end{array}$ \\
\hline
\end{tabular}

Fonte: ${ }^{1}$ Vannote et al. (1980); ${ }^{2}$ Adaptado de Fairfull \& Witheridge (2003); ${ }^{3}$ Lollo (1995); ${ }^{4}$ Beltrame (1994); ${ }^{5}$ Romero, Formiga \& Marcuzzo (2017).

\section{$4^{\text {a }}$ Etapa - Elaboração dos mapas}

Os mapas de altitude, relevo, rede de drenagem e distribuição espacial das nascentes foram elaborados com a ferramenta “novo compositor de impressão”, utilizando o sistema de coordenadas geográficas e o Datum WGS 84 com referência.

\section{Resultados e Discussão}

\subsection{Características geométricas}

A microbacia do rio Cutia tem área de $19,20 \mathrm{~km}^{2}$, perímetro de $24,79 \mathrm{~km}$, fator de forma de 0,33 , índice de circularidade de 0,39 e coeficiente de compacidade de 1,58, denotando forma alongada e baixa suscetibilidade a enchentes, do ponto de vista geométrico (Tabela 1). Uma bacia de formato alongado é menos suscetível a enchentes por ter menor probabilidade de ocorrência de precipitação pluviométrica simultânea em toda área, em comparação com bacias circulares de área equivalente (Vilella \& Mattos,1975). Este formato pode estar relacionado com a presença de Neossolos Quartzarênicos, que proporcionam boa capacidade de infiltração de água no solo, e consequentemente, menor dissecação do relevo (Calil et al., 2013). Em bacias localizadas nos estados de Minas Gerais e Rio de Janeiro, são observadas valores alongados e baixa suscetibilidade a enchentes, demonstrando que essas características também ocorrem em outros estados brasileiros (Cardoso et al., 2006; Tonello et al., 2006; Oliveira et al., 2010). A forma da bacia também influência o tempo de concentração, ou seja, o tempo necessário para que toda a bacia contribua para a saída da água após uma precipitação (Tonello et al., 2006). Nas bacias alongadas, os afluentes atingem o curso d'água principal em vários pontos ao longo do mesmo, diferentemente das bacias circulares, na qual a concentração do deflúvio 
se dá em um só ponto, diminuindo o tempo de concentração e aumentando as chances de ocorrência de enchentes (Villela \& Mattos, 1975)

\subsection{Características topográficas}

Os valores de altitude variam de 261 a 346 m, com valor médio de 300 m (Figura 2), e amplitude altimétrica de $85 \mathrm{~m}$. A altitude influencia a temperatura (Fritzons, Mantovani \& Aguiar, 2008; Fritzons, Where \& Mantovani, 2015; Fritzons, Mantovani \& Where, 2016), precipitação e a evaporação (Villela \& Mattos, 1975), e consequentemente, a distribuição espacial das espécies vegetais (Figueiredo et al., 2015). Mediante ao exposto, constata-se que existem mais de 20 espécies de interesse econômico que se adéquam a faixa de altitude da microbacia do rio Cutia, como exemplo tem-se (Bourke, 2010): 1) Abacate (Persea americana); 2) Abóbora (Cucurbita moschata); 3) Arroz (Oryza sativa); 4) Banana (Musa spp.); 5) Batata-doce (Ipomoea batatas); 6) Cacau (Theobroma cacao); 6) Café conilon (Coffea canephora var. robusta); 7) Cará (Dioscorea alata); 8) Feijão (Phaseolus vulgaris); 9) Inhame (Dioscorea esculenta); 10) Laranja (Citrus sinensis); 11) Limão (Citrus limon); 12) Mandioca (Manihot esculenta); 13) Melancia (Citrullus lanatus); 14) Melão (Cucumis melo); 15) Milho (Zea mays); 16) Pepino (Cucumis sativus); 17) Pimenta-do-reino (Piper nigrum); 18) Quiabo (Abelmoschus esculentus); 19) Seringueira (Hevea brasiliensis); 20) Taioba (Xanthosoma sagittifolium) e 21) Urucum (Bixa orellana). No entanto, por ocorrer predominância de solos da classe Neossolos Quartzarênicos (SEDAM, 2002), há a necessidade de práticas de manejo do solo como a calagem, manutenção da matéria orgânica do solo e aplicação de fertilizantes já que estes solos têm tendências a baixa fertilidade natural e baixa capacidade de troca de cátions.

A região tem as seguintes classes de relevo: plano (14,90\%), suave ondulado $(47,45 \%)$, ondulado $(34,58 \%)$, forte ondulado $(3,02 \%)$ e montanhoso (0,05\%) (Figura 3). Se considerar que o aumento da declividade eleva o risco de erosão hídrica, principalmente em solos arenosos e com ausência de vegetação (Bertoni \& Lombardi Neto, 2014), pode se inferir que as regiões mais suscetíveis às perdas de solo por erosão estão localizadas nos relevos montanhoso, forte ondulado e ondulado, respectivamente. Também é importante lembrar que os solos da microbacia são classificados como Neossolos Quartzarênicos (SEDAM, 2002), ou seja, tem texturas classificadas como areia ou areia franca em todos os horizontes até $150 \mathrm{~cm}$ de profundidade (no mínimo), a partir da superfície do solo ou até um contato lítico ou lítico fragmentário (Santos et al., 2018). A textura dos Neossolos Quartzarênicos limita ou até mesmo impossibilita a formação de agregados no solo, e reduz a capacidade de armazenamento de água, tornando-os altamente suscetíveis a erosão e escassez hídrica. Portanto, é recomendado a utilização de práticas integradas de manejo conservacionistas, para aumentar a capacidade de infiltração e o armazenamento de água no solo e, reduzir o escoamento superficial (Bertoni \& Lombardi Neto, 2014), optando-se principalmente por práticas vegetativas que favoreçam o aporte contínuo da matéria orgânica e cubram o solo durante o ano todo para formação e estabilização dos agregados (Wohlenberg et al., 2004).

A microbacia tem regiões classificadas como de baixa $(90,36 \%)$, moderada $(8,54 \%)$, alta $(0,94 \%)$, muito alta $(0,10 \%)$ e extremamente alta $(0,05 \%)$ influência na propagação de incêndios. Para reduzir os riscos de incêndios nas áreas mais críticas, deve-se evitar o uso de queimadas nas propriedades rurais e as margens das estradas. Estas informações são essenciais para os proprietários dos estabelecimentos agropecuários privados, Órgãos Ambientais e Corpo de Bombeiros, visto que, a identificação de risco de incêndio a partir de um SIG permite aos gestores planejar estrategicamente as atividades de prevenção a longo prazo (Paz et al., 2011).

Com relação a aptidão à mecanização agrícola, a microbacia tem 34,69, 39,32, 16,35, 6,56 e 3,07\% das áreas consideradas como extremamente apta, muito apta, apta, moderadamente apta e não apta a mecanização, respectivamente, confirmando que a maior parte da microbacia não tem limitações de declividade para utilização de mecanização agrícola, se considerar o cultivo de café como referência. No entanto, por se tratar de Neossolos Quartzarênicos o uso da mecanização se for adotada, 
deve ser cautelosa para evitar possíveis problemas de perda de solos por processos erosivos, visto que a região tem elevado índice pluviométrico.

Figura 2. Altitude da microbacia do rio Cutia, Amazônia Sul-Ocidental, Brasil.

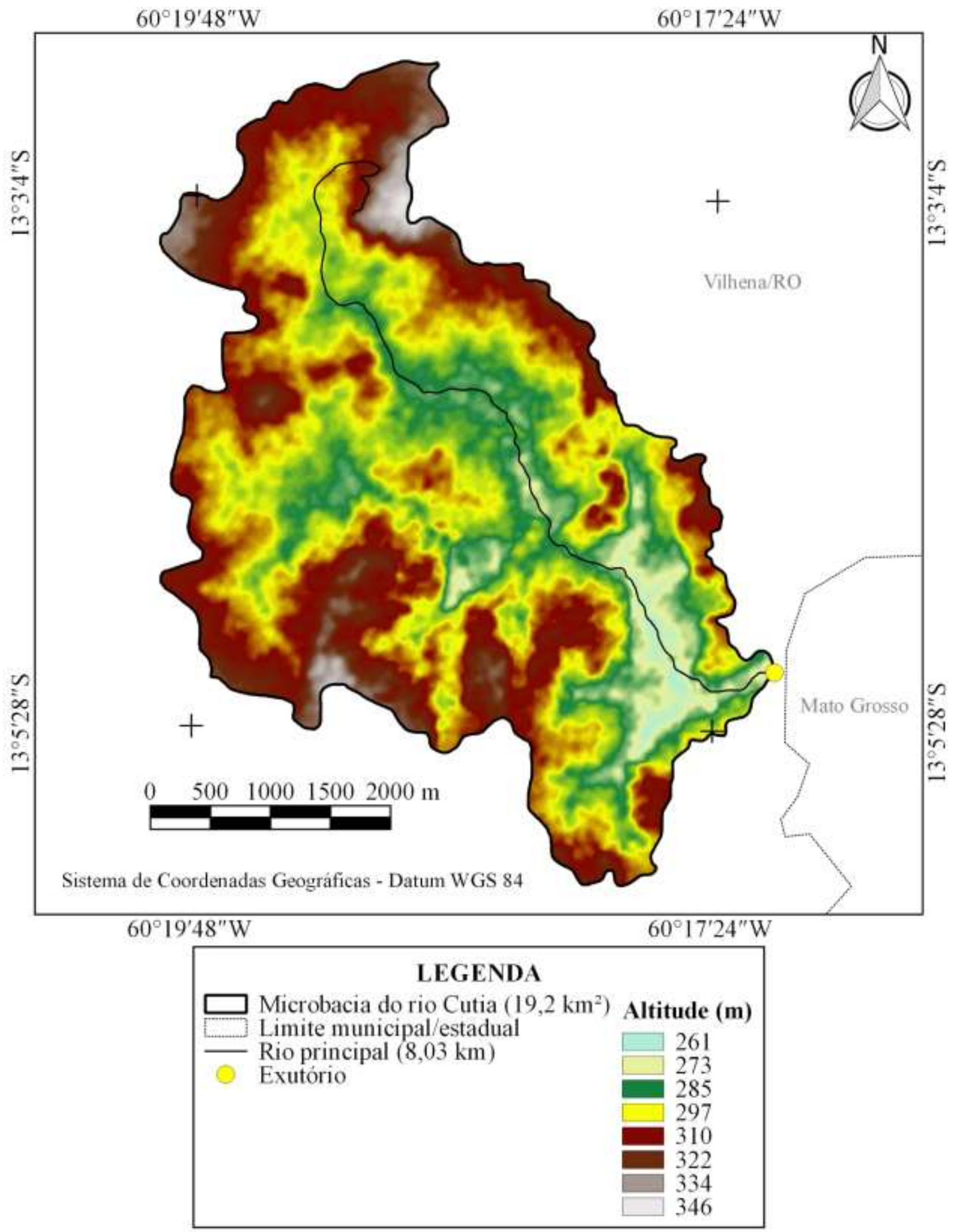

Fonte: Autores. 
Figura 3. Relevo da microbacia do rio Cutia, Amazônia Sul-Ocidental, Brasil.

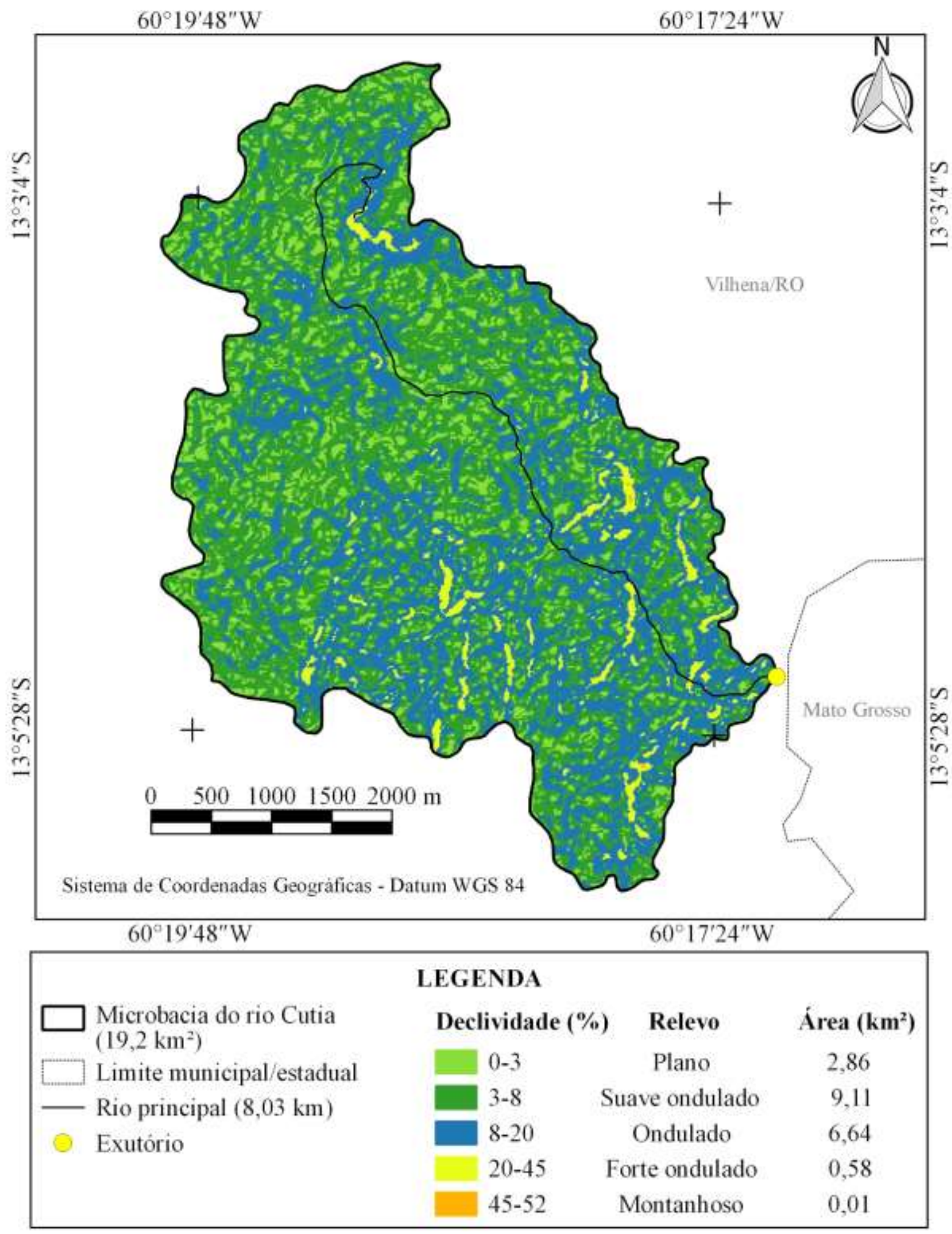

Fonte: Autores.

\section{Características hidrográficas}

A microbacia do rio Cutia tem rede de drenagem de 56,02 km, padrão dendrítico de $5^{\mathrm{a}}$ ordem (Figura 4), 7,81 nascentes $\mathrm{km}^{-2}$ (Figura 5), densidade de drenagem de 2,92 km km${ }^{-2}$, coeficiente de manutenção de $342,7 \mathrm{~m}^{2} \mathrm{~m}^{-1}$, índice de sinuosidade de $35,37 \%$ e tempo de concentração de $1,90 \mathrm{~h}$. 
Figura 4. Rede de drenagem e ordem dos rios na microbacia do rio Cutia, Amazônia Sul-Ocidental, Brasil.

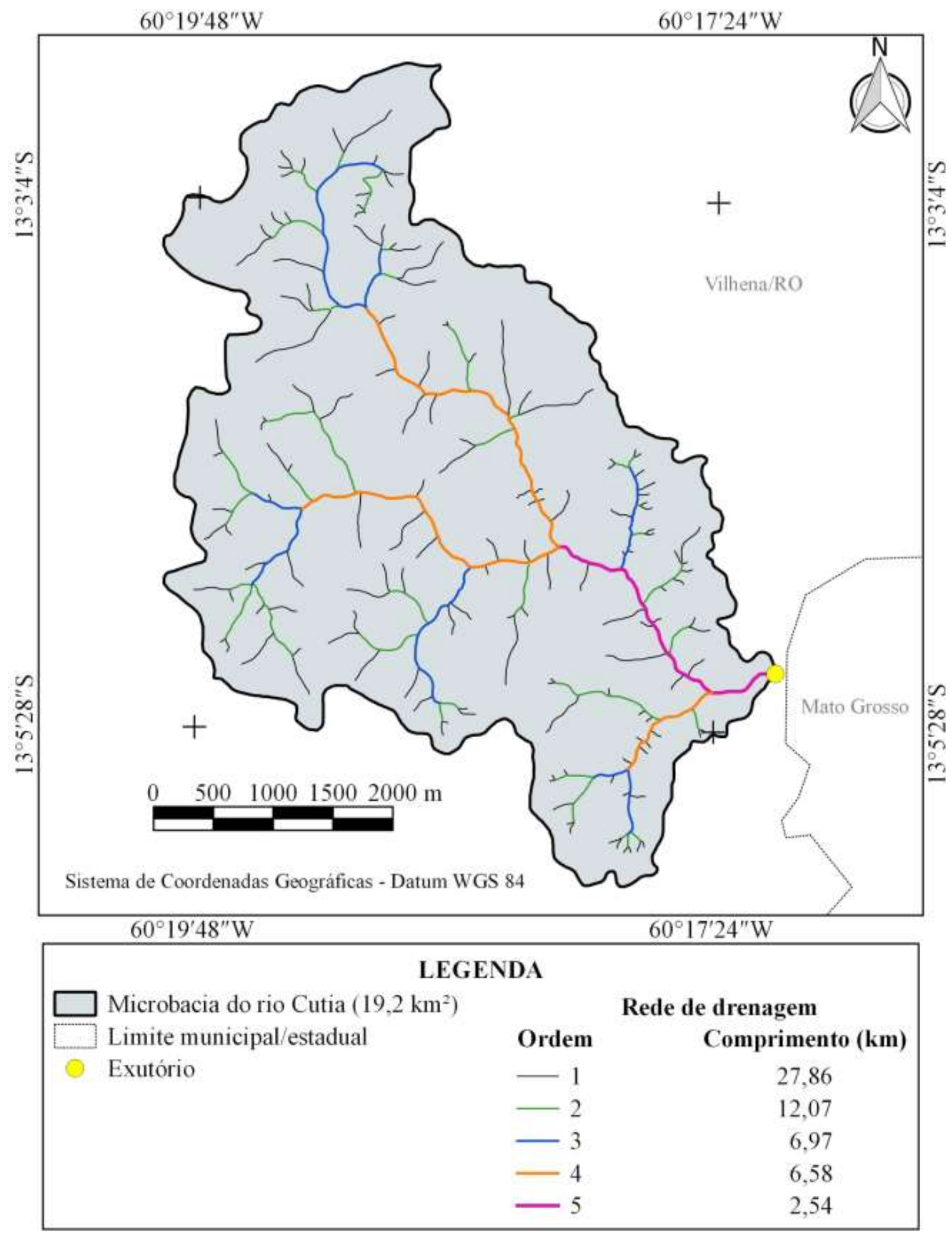

Fonte: Autores. 
Figura 5. Distribuição espacial das nascentes na microbacia do rio Cutia, Amazônia Sul-Ocidental, Brasil.

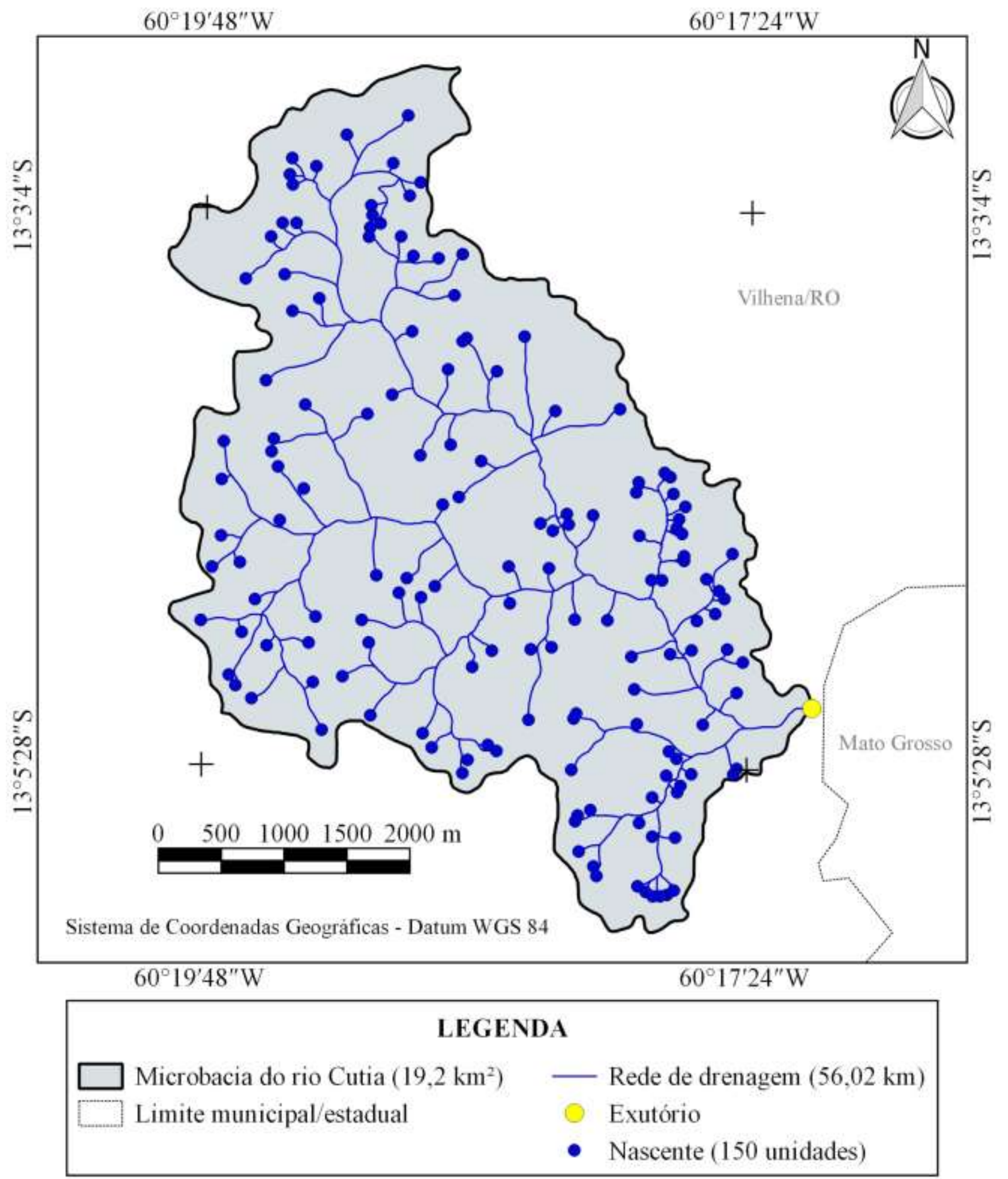

Fonte: Autores.

O padrão de drenagem dendrítico também é conhecido como arborescente, em função da semelhança com uma árvore (Smith 1943 apud Parvis, 1950), e denota boa distribuição espacial dos recursos hídricos, como pode ser observado na Figura 5. Este tipo de padrão de drenagem tem canais que se distribuem em todas as direções sobre a superfície e se unem formando 
ângulos agudos de graduações variadas, mas sem chegar ao ângulo reto (Christofoletti, 1980), e também foi observado nas microbacias dos rios Mutum (Souza et al., 2021), Paraíso (Lima et al., 2021) e Gavião (Donegá et al., 2021).

O número de ordens confirma a formação de um riacho médio (Tabela 3) com sistema de drenagem complexo (Horwitz, 1978), e elevadas condições para habitação de peixes (Tabela 3). Em estudo realizado por Vannote et al. (1980), em rios de 1 a $12^{\circ}$ ordem, constatou-se que a diversidade biótica foi maior em rios de $4^{\mathrm{a}}$ a $5^{\mathrm{a}}$ ordem. Com base nestas informações recomendase estudos mais detalhados a respeito da complexidade do ecossistema aquático, para elaboração de estratégias que visem a manutenção dos recursos hídricos, levando em consideração quantidade e qualidade hídrica, e, consequentemente, a conservação das espécies de peixes nativos da região.

As densidades de nascentes e drenagem são consideradas altas (Tabela 3). Estas características sugerem que a microbacia tem elevada capacidade para gerar novos cursos d'água (Christofoletti, 1969) e boa capacidade de drenagem (Villela \& Mattos, 1975). Ambas densidades estão associadas a uma combinação de fatores, incluindo declividade (Vendruscolo et al., 2020a; Vendruscolo et al., 2020b), textura e cobertura do solo (Bertoni \& Lombardi Neto, 2014), visto que o aumento da declividade, a presença de solos arenosos e ausência de vegetação, favorecem a erosão hídrica e a formação de canais em sulco. Este tipo de erosão pode evoluir com o tempo, passando para o tipo ravina e em seguida para voçoroca, até alcançar o lençol freático (Guerra, 1997), originando novos canais e novas nascentes.

Ao considerar a presença de Neossolos Quartzarênicos na microbacia, constata-se a necessidade de manter a vegetação nativa da zona ripária, com o intuito de evitar o colapso das margens e assoreamentos dos recursos hídricos, e em outras posições do relevo, para garantir a disponibilidade e qualidade da água, e aporte de matéria orgânica ao solo a partir de restos vegetais para auxiliar no processo de agregação do solo. A vegetação nativa desempenha funções eco-hidrológicas distintas (Tambosi et al., 2015), dependendo de sua localização na paisagem: abastecimento do lençol freático (topos de morros), contenção de processos erosivos (encostas), filtro de poluentes e contaminantes (zona ripária) e funções auxiliares (intervales). Neste contexto, recomenda-se estudos sobre a análise temporal e espacial de cobertura do solo, para entender a dinâmica de uso e ocupação do solo, selecionar práticas de manejo conservacionista do solo nos sistemas agropecuários e delimitar áreas prioritárias para a manutenção da vegetação nativa. Além disso, na região da microbacia, ocorrem manchas de cerrado (IBGE, 2012), um ecossistema de grande importância para a biodiversidade do estado de Rondônia e que tem sido continuamente transformado em áreas agrícolas (Sccoti \& Sccoti, 2019).

O coeficiente de manutenção fornece a área mínima necessária para a manutenção de um metro de canal de escoamento, de modo que são necessários $342,7 \mathrm{~m}^{2}$ de área para manter $1 \mathrm{~m}$ de rio. Esse valor é inferior aos observados nas microbacias dos rios Águas Claras (366,5 $\mathrm{m}^{2} \mathrm{~m}^{-1}$ ) (Santos et al., 2021), Mutum (499,4 $\mathrm{m}^{2} \mathrm{~m}^{-1}$ ) (Souza et al., 2021), Jacuri $\left(1.102,9 \mathrm{~m}^{2} \mathrm{~m}^{-1}\right.$ ) (Panza et al., 2020) e Gavião (1.250,00 $\mathrm{m}^{2} \mathrm{~m}^{-1}$ ) (Donegá et al., 2021). Portanto, a microbacia do rio Cutia necessita de uma área menor para manutenção dos recursos hídricos, em comparação com as microbacias citadas.

O valor do índice de sinuosidade confirma a presença de um canal divagante (Tabela 3), assemelhando-se ao canal principal da microbacia Águas Claras (Santos et al., 2021), e diferenciando-se dos canais principais das microbacias Três Galhos (Silva et al., 2021), Paraíso (Lima et al., 2021) e Tamarupá (Vendruscolo et al., 2021b), os quais apresentam canal sinuoso. Este parâmetro é um fator controlador do escoamento (Villela \& Mattos, 1975), e interfere diretamente no tempo de concentração. Canais divagantes tendem a acumular sedimentos, e esse acúmulo de sedimentos na parte interna dos meandros é essencial para a formação de praias de água doce, utilizadas por algumas espécies de tartarugas como berçário natural na região amazônica (Ferreira Júnior, 2009). Portanto, a microbacia do rio Cutia possui potencial para estudos direcionados à identificação de berçários naturais e proteção da fauna silvestre. 
Em relação ao tempo de concentração, constatou-se que são necessários $1,90 \mathrm{~h}$ para que a água percorra 8,03 km, denotando um fluxo hídrico com velocidade média de $4,23 \mathrm{~km} \mathrm{~h}^{-1}$. O tempo de concentração encontrado é inferior aos encontrados em outras microbacias, a exemplo: São Jorge 3,63 h (adaptado de Pacheco et al., 2020), Médio Rio Escondido 4,46 h (adaptado de Vendruscolo et al., 2020b) e Alto Rio Escondido 5,02 h (adaptado de Vendruscolo et al., 2020a). Estas diferenças no tempo de concentração estão associadas ao comprimento do canal, visto que segundo os autores, as microbacias citadas apresentam canais mais extensos, com comprimentos de 16,07,24,05 e 27,93 km, respectivamente. Também, observa-se que o tempo de concentração é baixo quando comparado ao tempo de precipitação da região (Santos Neto, 2014). Portanto, toda a área da microbacia pode contribuir simultaneamente para a vazão do rio, potencializando os riscos de enchentes, mesmo que a bacia tenha sido classificada geometricamente de baixa probabilidade.

\section{Conclusão}

A microbacia do rio Cutia tem área de 19,20 km² de área, perímetro de 24,79 km, forma alongada, baixa suscetibilidade a enchentes, altitudes de 261 a 346 m, predominância de relevo suave ondulado (47,45\%), 90,36\% da área apta a extremamente apta a mecanização agrícola e com baixa influência na propagação de incêndios, rede de drenagem com padrão dendrítico, $5^{\mathrm{a}}$ ordem de drenagem, alta densidade de nascentes, alta densidade de drenagem, coeficiente de manutenção de $342,7 \mathrm{~m}^{2} \mathrm{~m}^{-1}$, canal principal divagante e baixo tempo de concentração.

As características da paisagem confirmam o potencial para implantação de sistemas agropecuários, inclusive mecanizado em boa parte da microbacia. No entanto, são recomendadas práticas de manejo conservacionistas do solo e da água, para mitigar problemas com enchentes e o impacto negativo dos sistemas agropecuários sobre os recursos naturais. Também são recomendados estudos sobre a distribuição espacial e temporal da vegetação nativa, principalmente nas áreas protegidas por lei (reservas legais e áreas de preservação permanente), para auxiliar na delimitação de áreas prioritárias para a conservação dos recursos hídricos e para a conservação de ecossistemas florestais ameaçados no Estado.

\section{Referências}

Alvares, C. A., Stape, J. L., Sentelhas, P. C., Gonçalves, L. M., \& Sparovek, G. (2013). Köppen's climate classification map for Brazil. Meteorologische Zeitschrift, 22(6), 711-728. 10.1127/0941-2948/2013/0507.

ASF - Alaska Satellite Facility (2017). Imagem altimétrica. https://www.asf.alaska.edu/

Beltrame, A. V. (1994). Diagnóstico do meio ambiente físico de bacias hidrográficas: modelo de aplicação. Florianópolis: Universidade Federal de Santa Catarina.

Bertoni, J., \& Lombardi Neto, F. (2014). Conservação do solo. (9a ed.), Ícone.

Bourke, R. M. (2010). Altitudinal limits of 230 economic crop species in Papua New Guinea. In: Haberle, S. G., Stevenson, J., \& Prebble, M. (eds). Altered Ecologies: Fire, Climate and Human Influence on Terrestrial Landscapes. ANU E-Press.

Calil, P. M., Oliveira, L. F. C., Kliemann, H. J., \& Oliveira. V. A. (2013). Caracterização geomorfométrica e do uso do solo da bacia hidrográfica do Alto Meia Ponte, Goiás. Revista Brasileira de Engenharia Agrícola e Ambiental, 16(4): 433-442. 10.1590/S1415-43662012000400014

Cardoso, C. A. C, Dias, H. C. T., Soares, C. P. B., \& Martins, S. V. (2006). Caracterização morfométrica da bacia hidrográfica do rio Debossan, Nova Friburgo, RJ. Revista Árvore, 30(2), 241-248. 10.1590/S0100-67622006000200011

Cavalheiro, W. C. S., \& Vendruscolo, J. (2019). Importância de estudos em bacias hidrográficas para o manejo sustentável dos recursos hídricos em Rondônia. Revista Geográfica Venezolana, especial, 256-264.

Christofoletti, A. (1969). Análise morfométrica de bacias hidrográficas. Notícia Geomorfológica, 18(9), 35-64.

Christofoletti, A. (1980). Geomorfologia. Edgard Blucher.

Donegá, M. V. B., Souza, T. W. S., Lima, M. M., Panza, M. R., Pacheco, F. M. P., Saraiva, J. G., Cavalheiro, W. C. S., \& Vendruscolo, J. (2021). Caracterização hidrogeomorfométrica da microbacia do rio Gavião, Amazônia Ocidental, Brasil. Research, Society and Development, 10(1), 1-14. 10.33448/rsd-v10i1.11844

Fairfull, S., \& Witheridge, G. (2003). Why do Fish Need to Cross the Road? Fish Passage Requirements for Waterway Crossings. NSW Fisheries. 
Ferreira Junior, P. D. (2009). Aspectos ecológicos da determinação sexual em tartarugas. Acta Amazônica, 39(1), 139-154. 10.1590/S0044-59672009000100014

Figueiredo, S. M. M., Venticinque, E. M., Figueiredo, E. O., \& Ferreira, E. J. L. (2015). Predição da distribuição de espécies florestais usando variáveis topográficas e de índice de vegetação no leste do Acre, Brasil. Acta Amazonica, 45(2), 167-174. 10.1590/1809-4392201402834

Florenzano, T. G., Lima, S. F. S. \& Moraes, E. C. (2011). Formação de professores em geotecnologia por meio de ensino a distância. Educar em Revista, 40, 69-84. 10.1590/S0104-40602011000200006

Franca, R. R. (2015). Climatologia das chuvas em Rondônia - período 1981-2011. Revista Geografias, 11(1), 44-58.

Fritzsons, E., Mantovani, L. E., \& Aguiar, A. V. (2008). Relação entre altitude e temperatura: uma contribuição ao zoneamento climático no estado do Paraná. Revista de Estudos Ambientais, 10(1): 49-64, 2008. 10.7867/1983-1501.2008v10n1p49-64

Fritzsons, E., Mantovani, L. E., \& Wrege, M. S. (2016). Relação entre altitude e temperatura: uma contribuição ao zoneamento climático no estado de Santa Catarina, Brasil. Revista Brasileira de Climatologia, 12(8), 80-92, 2016. 10.5380/abclima.v18i0.39471

Fritzsons, E., Wrege, M. S., \& Mantovani, L. E. (2015). Altitude e temperatura: estudo do gradiente térmico no Rio Grande do Sul. Revista Brasileira de Climatologia, 11(16), 108-119, 2015. 10.5380/abclima.v16i0.39665

Guerra, A. J. T. (1997). Ravinas: processo de formação e desenvolvimento. Anuário do Instituto de Geociências, 20, 9-26.

Höfig, P., \& Araujo-Junior, C. F. (2015). Classes de declividade do terreno e potencial para mecanização no estado do Paraná. Coffee Science, 10(2), 195-203.

Horton, R. E. (1932). Drainage basin characteristics. Transactions, American Geophysical Union, 13(1), 350-361.

Horwitz, R. J. (1978). Temporal variability patterns and the distributional patterns of stream fishes. Ecological Monographs, 48(3), 307-321. 10.2307/2937233

IBGE - Instituto Brasileiro de Geografia e Estatística. (2012). Manual técnico da vegetação brasileira. IBGE.

INCRA - Instituto Nacional de Colonização e Reforma Agrária (2018). Acervo fundiário. http://acervofundiario.incra.gov.br/acervo/acv.php.

Jucá, S. C. S., Carvalho, P. C. M., \& Aguiar Júnior, J. S. (2006). A relevância dos sistemas de informação geográfica no desenvolvimento das energias renováveis.Ciências \& Cognição, 9, 131-136.

Lima Júnior, J. C., Vieira, W. L., Macêdo, K. G., Souza, S. A., \& Nascimento, F. A. L. (2012). Determinação das características morfométricas da sub-bacia do Riacho Madeira Cortada, Quixelô, CE. Anais do Congresso Norte-Nordeste de Pesquisa e Inovação. Palmas, TO, Brasil, 7.

Lima, M. M., Donegá, M. V. B., Souza, T. W. S., Panza, M. R., Pacheco, F. M. P., Cavalheiro, W. C. S., Hara, F. A. S., \& Vendruscolo, J. (2021). Hidrogeomorfometria da microbacia do rio Paraíso: informações para auxiliar o manejo dos recursos naturais na Amazônia Ocidental. Research, Society and Development, 10(3), 1-20. 10.33448/rsd-v10i3.13367

Lollo, J. A. (1995). O uso da técnica de avaliação do terreno no processo de elaboração do mapeamento geotécnico: sistematização e aplicação na quadrícula de Campinas. (Tese de Doutorado). Universidade de São Paulo, São Carlos, SP, Brasil.

Moreira, M. A. (2001). Fundamentos do Sensoriamento Remoto e Metodologias de Aplicação. São José dos Campos: Instituto Nacional de Pesquisas Espaciais.

Oliveira, P. T. S., Sobrinho, T. A., Steffen, J. L., \& Rodrigues, D. B. B. (2010). Caracterização morfométrica de bacias hidrográficas através de dados SRTM. Revista Brasileira de Engenharia Agrícola e Ambiental, 14(8), 819-82. 10.1590/\$1415-43662010000800005

Pacheco, F. M. P., Vendruscolo, J., Ramos, F. H., Rodrigues, A. A. M., Cavalheiro, W. C. S., Hara, F. A. S., Rocha, K. J., \& Silva, G. N. (2020). Caracterização hidrogeomorfométrica da microbacia do rio São Jorge, Rondônia, Brasil. Brazilian Journal of Development, 6(1), 4219-4236. 10.34117/bjdv6n1-301

Panza, M. R., Donegá, M. V. B., Pacheco, F. M. P., Nagao, E. O., Hara, F. A. S., Cavalheiro, W. C. S., \& Vendruscolo, J. (2020). Características da paisagem para manejo dos recursos naturais na microbacia do Rio Jacuri, Amazônia Ocidental, Brasil. Brazilian Journal of Development, 6(12), 101532-101558. $10.34117 /$ bjdv6n12-592

Parvis, M. (1950). Drainage pattern significance in airphoto identification of soils and bedrocks. Photogrammetric Engineering, 16, 387-408.

Paz, S., Carmel, Y., Jahshan, F., Shoshany, M. (2011). Post-fire analysis of pre-fire mapping of fire- risk: a recent case study from Mt. Carmel (Israel). Forest Ecology and Management, 262, 1184-1188. 10.1016/j.foreco.2011.06.011

Pereira, A. S., Shitsuka, D. M., Parreira, F. J., \& Shitsuka, R. (2018). Metodologia da Pesquisa Científica. Santa Maria-RS: Universidade Federal de Santa Maria.

Ribeiro, L., Koproski, L. P., Stolle, L., Lingnau, C., Soares, R. V., \& Batista, A. C. (2008). Zoneamento de riscos de incêndios florestais para a Fazenda Experimental do Canguiri, Pinhais (PR). Floresta, 38(3), 561-572. 10.5380/rf.v38i3.12430

Romero, V., Formiga, K. T. M., \& Marcuzzo, F. F. N. (2017). Estudo hidromorfológico de bacia hidrográfica urbana em Goiânia/GO. Ciência e Natura, 39(2), 320-340. 10.5902/2179460X26411

Santos, A. A., Silva, E. C., Cardoso, L. A. P., Praia, W. M., Hara, F. A. S., Cavalheiro, W. C. S., \& Vendruscolo, J. (2021). Características hidrogeomorfométricas da microbacia do rio Águas Claras, Amazônia Ocidental, Brasil. Research, Society and Development, 10(3), 1-16. 10.33448/rsd-v10i3.13363

Santos, A. M., Targa, M. S., Batista, G. T., \& Dias, N. W. (2012). Análise morfométrica das sub-bacias hidrográficas Perdizes e Fojo no município de Campos do Jordão, SP, Brasil. Revista Ambiente \& Água, 7(3), 195-211. 10.4136/1980-993X 
Santos, H. G., Jacomine, P. K. T., Anjos, L. H. C., Oliveira, V. Á., Lumbreras, J. F., Coelho, M. R., Almeida, J. A., Araújo Filho, J. C., Oliveira, J. B., \& Cunha, T. J. F. (2018). Sistema Brasileiro de Classificação de Solos. Embrapa Solos.

Santos Neto, L. A. (2014). Variabilidade da precipitação horária em Porto Velho-RO e suas tendências anuais e sazonais. (Dissertação de Mestrado). Universidade Federal de Rondônia.

Santos, R. D., Lemos, R. C., Santos, H. G., Ker, J. C., Anjos, L. H. C., \& Shimizu, S. H. (2013). Manual de descrição e coleta de solo no campo. Sociedade Brasileira de Ciência do Solo.

Sccoti. M.S.V., \& Sccoti, A.A.V. (2019). A vegetação natural do estado de Rondônia. In: Stachiw, R. (Org.) Nossa terra, nossa gente: uma contribuição à história e geográfia de Rondônia. Gráfica e Editora Imediata.

SEDAM - Secretaria de Estado do Desenvolvimento Ambiental (2002). Atlas Geoambiental de Rondônia. SEDAM.

Silva, E. C., Praia, W. M., Santos, A. S., Cardoso, L. A. P., Hara, F. A. S., Cavalheiro, W. C. S., \& Vendruscolo, J. (2021). Características hidrogeomorfométricas da microbacia Três Galhos, Amazônia Ocidental, Brasil. Research, Society and Development, 10(2), 1-17, 2021. 10.33448/rsd-v10i2.12408

Silva, Q. D. (2012). Mapeamento geomorfológico da Ilha do Maranhão. (Tese de Doutorado). Universidade Estadual Paulista.

Soares, G. S., Santos Júnior, N. R. F., Mira, S. F., Moreto, R. F., Cavalheiro, W. C. S., Vendruscolo, J., \& Rosa, D. M. (2019). Uso de plataforma SIG na caracterização morfométrica da microbacia do rio Santa Teresinha, Amazônia Ocidental, Brasil. Revista Geográfica Venezolana, 84-95. http://www.saber.ula.ve/handle/123456789/46157

Souza, T. W. S., Lima, M. M., Saraiva, J. G., Pacheco, F. M. P., Donegá, M. V. B., Panza, M. R., Cavalheiro, W. C. S., \& Vendruscolo, J. (2021). Análise hidrogeomorfométrica da microbacia do rio Mutum: informações para auxiliar o manejo de recursos hídricos na Amazônia Ocidental. Research, Society and Development, 10(2), 1-17. 10.33448/rsd-v10i2.12448

Strahler, A. N. (1954). Quantitative geomorphology of erosional landscapes. XIX International Geologic Congress, 13(15), $341-354$.

Tambosi, L., Vidal, M. M., Ferraz, S. F. B., \& Metzger, J. P. (2015). Funções eco-hidrológicas das florestas nativas e o Código Florestal. Estudos Avançados, 29(84), 151-162. 10.1590/S0103-40142015000200010

Targa, M. S., Batista, G. T., Diniz, H. D., Dias, N. W., \& Matos, F. C. (2012). Urbanização e escoamento superficial na bacia hidrográfica do Igarapé Tucunduba, Belém, PA, Brasil. Revista Ambiente \& Água, 7(2), 120-142. 10.4136/1980-993X

Tonello, K. C., Dias, H. C. T., Souza, A. L., Ribeiro, C. A. A. S., \& Leite, F. P. (2006). Morfometria da bacia hidrográfica da Cachoeira das Pombas, Guanhães - MG. Revista Árvore, 30(5), 849-857. 10.1590/S0100-67622006000500019

Vannote, R. L., Minshall, G. W., Cummins, K. W., Sedell, J. R., \& Cushing, C. E. (1980). The river continuum concept. Canadian Journal of Fisheries and Aquatic Sciences, 37, 130-137.

Vendruscolo, J., Araujo, M. G. S., Ferreira, L. R., Rosa, D. M., \& Hara, F. A. S. (2021b). O uso de geotecnologias na caracterização geométrica, topográfica e hidrográfica da microbacia do rio Tamarupá, Amazônia Ocidental, Brasil. Brazilian Journal of Development, 7(1), 4245-4264. 10.34117/bjdv7n1-286

Vendruscolo, J., Boone, N. R. V., Moreto, R. F., Santos Júnior, N. R. F., Soares, G. S., Lima, A. C. R., Cavalheiro, W. C. S., Scotti, M. S. V., Maia, E., \& Hara, F. A. S. (2021a). Características da paisagem da sub-bacia do rio Escondido, Amazônia sul-ocidental, Brasil. Research, Society and Development, 10(3), 1-25. $10.33448 /$ rsd-v10i3.13253

Vendruscolo, J., Pacheco, F. M. P., Ramos, H. F., Cavalheiro, W. C. S., \& Rodrigues. A. A. M. (2020a). Hidrogeomorfometria da microbacia Alto Rio Escondido: informações para auxiliar o manejo dos recursos naturais na Amazônia ocidental. Brazilian Journal of Development, 6(3), 9709-9730. 10.34117/bjdv6n3-011

Vendruscolo, J., Pacheco, F. M. P., Rodrigues, A. A. M., Ramos, H. F., Rosa, D. M., \& Cavalheiro, W. C. S. (2020b). Características morfométricas da microbacia do Médio Rio Escondido, Amazônia Ocidental, Brasil. Brazilian Journal of Development, 6(1), 565-585. 10.34117/bjdv6n1-040

Villela, S. M., \& Mattos, A. (1975). Hidrologia aplicada. McGraw-Hill.

Wohlenberg, E. V., Reichert, J. M., Reinert, D. J., \& Blume, E. (2004). Dinâmica da agregação de um solo franco-arenoso em cinco sistemas de culturas em rotação e em sucessão. Revista Brasileira de Ciência do Solo. 28(5), 891-900. 10.1590/S0100-06832004000500011 